\title{
Traffic Dynamics in Virtual Routing Multi Topology System
}

\author{
K. Chennakeshavulu, K Rusheek, K Srihitha \\ Hyderabad
}

\begin{abstract}
Providing a better performance is the key in IP network systems.An Adaptive Multipath Routing(AMR) system is introduced to handle the unpredicted traffic dynamics. The proposed system consists of Weight Computation component that sets the link weights and producing the maximum paths in the virtual routing multi topology. Based on the produced paths, an adaptive Traffic Splitting mechanism performing the traffic splitting across for every destination in the network within a short period. The system is using the Multiple Routing Configurations method to handle the node and link failures in the network, allowing packet forwarding to carry on pre-configured alternative next-hops immediately after the recognition of the failure .The proposed mechanism giving the better quality of service and network performance.
\end{abstract}

Keywords: $A M R, A T C, I G P, T E$

\section{Introduction}

Traffic Engineering aims at optimizing the performance of operational in IGP networks. The existing mechanisms cannot handle the unpredicted dynamic traffics efficiently, allowing only the static traffic delivery. Changing the link weights will cause the network reconvergence problem so that re computation of dynamic/online routing is to be considered. Instead of a physical connection between the devices, virtual network concept is implemented using the network virtualization method. Each having its own IGP(interior gateway routing protocol)link weight settings.IGP protocols underlies the platform to support the coincide of multi virtual paths in between the two end points source and destinations.

The proposed an adaptive multipath routing system is based on network virtualization method to cope the unpredicted traffic dynamics and proving the better quality of network performance. The idea is that provisioning of diverse routing paths in offline and for a dynamic load balancing spreading the traffic in online. Based on the link weights the user traffic is assigned to different virtual topologies. The physical network topology is an input to the IGP link weight optimization. The figurel explains how link weight configurations can be assigned to maximum path diversity across virtual routed topologies.

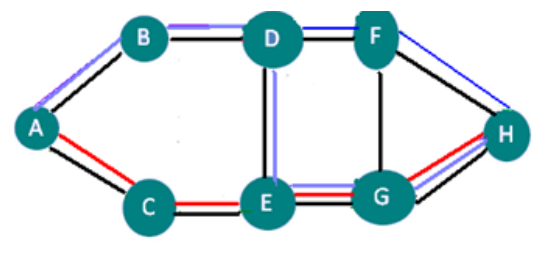

VRT1 FIG1 VRT2 VRT3

For every source,destination S-D pair offline configuration is computing the link weights to provide maximum path diversity.After configuring the link weights, an adaptive traffic algorithm performing the traffic splitting ratio adjustment to handle load balancing within the short time scale.For example the link weight between DE is highly loaded in VRT2 the path is shifted to other path DF across VRT3.the original path at D is shifted to F with the blue color.This is done at A node by adjusting the traffic splitting ratio across the three Virtual Routing Topologies. The ultimate task is to adjusting the traffic assignment at every source nodes in reactions to the traffic conditions.

Adaptive Multipath Routing System consisting of weight computation component and Traffic Splitting component. Weight Computation component takes an input from the physical network topology and trying to produce maximum path diversity across multiple virtual routing topology through the settings of link weights.Acording to the traffic conditions the traffic splitting component performing the traffic splitting operations across the routing topologies within short time scale.

\section{SYSTEM Framework}

The FIG 2 describes how the traffic demands and performance objectives are depends on several things while assigning the link weights. The Traffic Conditions Data Base maintaining the information about elements of the network and current traffic conditions, which are used to perform other operational tasks. Changing of link weights depends on how the IGP configuration is affecting to the traffic flow. 


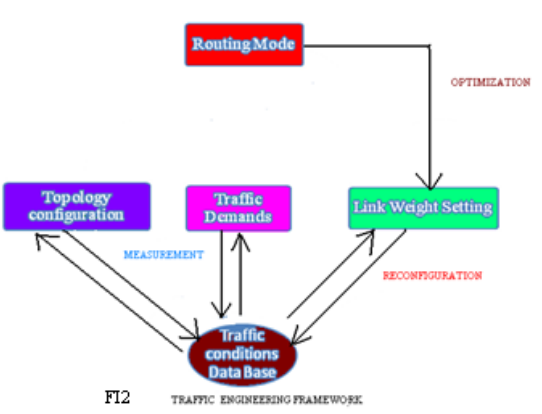

To provide the better performance and reliability conditions the best changing of link weights are to be considered.An efficient Adaptive Traffic Control algoritham is used to compute the link weights to satisfy these conditions.Adaptive traffic control computing the new traffic splitting ratios across individual virtual routing topologies for reassigning the traffic the diverse paths between each source and destination pair.Traffic Engineering manager is handling these functionalities by periodically gathering the up to date monitoring traffic conditions of the network. The new splitting ratios are then configured by the TE manager to individual source nodes.

Path diversity:The fundamental issue of an offline link weight optimization is to determine the path diversity across the multiple diverse paths. The scenarios to determining the path diversity

- End to end highly disjoint diverse paths are available for some source destination pairs, while some other pairs individual paths are completely overlapping with each other

- None of the source destination pairs are disjoint paths.

In the first case if a link which is shared by all paths becomes congested, its load cannot be improved by adjusting the traffic splitting ratios at the respected source nodes.Achieving the balanced path diversity across all source destination pairs in the second case. We define the Full Degree of Involvement (FDoI) binary metric to evaluate the overall path diversity.The FDoI value is set to 1 with respect to source destination pair if this link is shared by the shortest paths across all VRTs for that source destination pairs. Otherwise the FDoI value is set to 0. if the sum is equal to 0 then no critical link is formed.

Network monitoring is responsible for collecting the up to date traffic situations to performing the ATC operations. The monitoring agent at every node monitoring

- The traffic volume originated by the local users towards other.

-the directly attached inter-pop links utilization.

The monitoring agent is gathering the traffic volume from all the access routers. The TE manager is polling the individual monitoring agents and collecting the monitored traffic volume, link utilizations in a periodic fashion. The TE manager is using these statistics to update the maintained traffic in the Traffic Conditions Data Base and computing the traffic splitting ratios for the next interval.

\section{AdaPTIVE Traffic CONTROL:}

The objective of ATC is to minimizing the maximum link utilizations in the network. To achieve this goal an efficient algorithm is used applied to adjusting the traffic splitting ratio at individual pop source nodes. The following operations are performed in a periodic fashion

- Measuring the incoming traffic volume and network load for the current interval.

- Computing the new splitting ratios at individual source nodes based on the splitting ratio configuration in the previous interval.

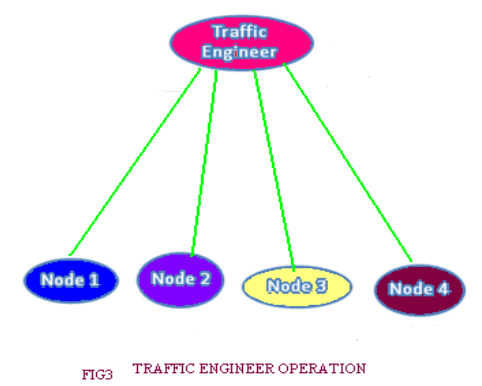

TE manager is maintaining the traffic conditions data base to the network status. The traffic conditions data base consisting of link list and source destination pair list.The link list is maintaining the list of entries for each 
network links. The source destination pair list consisting of a list of entries each for specific source destination pair with the most recently measured traffic volume from source to destination. For each ATC interval, traffic conditions data base is updated. Based on the up to date data maintained on the traffic conditions data base, the ATC operation is performed.

The procedure to the ATC algorithm is as follows

- Identify the maximum utilization link $1_{\max }$ the network. This is done by observing the link list in the traffic conditions data base

- The set of source destination pairs whose traffic flows are routed through the maximum utilization link $1_{\max }$ in $\mathrm{FDoI}=0$, compute the new traffic splitting ratio for each one at a time until the feasible traffic flow is identified.

- After a feasible one is identified, accept the new splitting ratio adjustment. Increment the counter by one and go to step 1 if the maximum $\mathrm{k}$ iterations have not been reached. If no feasible traffic flow is exists stop the procedure and configure the latest resulting values in the source destination pair list.

It is an important issue to viewing the sensitive information only an authorized users. To provide a confidentiality, e-signature solution is designed to handle an attack where a user gets between the source and destination of information. An adaptive multipath routing system is using the generating of an Electronic signature to authenticate a message which is based on MAC algorithm. E signature is verifying a specific user was present and transforming the information in a network.

\section{Proposed System}

Analyzing the list of packets: An adaptive multipath routing system monitoring the different things with wireshark to provide a better performance and quality of service. The system is checking if there are any unwanted packets are being sending or receiving from the node. Sending the repeated and unwanted data is affecting the network performance. If such a particulers is observed in the traffic conditions data base, blocking the user who is transforming unwanted data by observing the past transmitted data. Transmit in a different path if the necessary data is transforming between the two users in terms of required operation. The Traffic Engineer is responsible to find out what program is taking up all network traffic and affecting the network performance and monitor how often a program uses the network. An adaptive multipath routing system is the key to the future work is not a restriction with number of topologies. The system should solve the network problems..

\section{Handling the node and link failures.}

The system is Proposing a method Multiple Routing Configurations to handling the node and link failures in a network which is allowing recovery in the range of milliseconds. Multiple Routing Configurations are allowing packet forwarding to carry on pre-configured alternative next-hops immediately after the recognition of the failure. The main objective of multiple routing configurations is to use the network graph and associated link weights to generate a set of back up configurations. When a failure is occurred backup routing configurations are used to and thus we can select the link weights in the backup configurations. The dissemination times of failures with re computation of forwarding table defining the minimum time for responding to the failures. It may be preferable that convergence is handled by backup paths with fast failure mechanisms in the network element. Multiple Routing configurations will give the potentially backup paths that are longer than the optimal paths.

\section{Conclusion}

An Adaptive Multipath Routing system handling the unpredicted traffic dynamics in a network. Using the weight computation component to compute the link weight settings and to produce maximum path diversity across the virtual routing multi topology. Based on the produced path diversity Traffic splitting component is adjusting the traffic dynamics by splitting the traffic volume at every individual virtual routing topologies. Multiple Routing Configurations method to handle the node and link failures in the network, allowing packet forwarding to carry on pre-configured alternative next-hops immediately after the recognition of the failure .The proposed mechanism giving the better quality of service and network performance. 


\section{References}

[1]. N. Wang, K-H. Ho, and G. Pavlou, “Adaptive Multi- topology IGP Based Traffic Engineering with Near-Opti- mal Performance," Proc. IFIP Networking 2008.

[2]. S. Uhlig et al., "Providing Public Intradomain Traffic Matrices to the Research Community," ACM Sigcomm Comp. Commun. Rev. (CCR), vol. 36, no. 1, Jan. 2006, pp. 83-86.

[3]. B. Fortz and M. Thorup, “Optimizing OSPF/IS-IS Weights in a Changing World,” IEEE JSAC, vol. 20, no. 4, May 2002, pp. 75667.

[4]. D. Xu, M. Chiang, and J. Rexford, "Link-State Routing With Hop-By-Hop Forwarding Can Achieve OptimalTraffic Engineering," Proc. IEEE INFOCOM, Apr. 2008.

[5]. M. Caesar et al., "Dynamic Route Computation Considered Harmful," ACM Comp. Commun. Rev. (CCR), vol.40, no. 2, Apr. 2010, pp. 66-71.

[6]. Wang, J., et al.: Edge-based Traffic Engineering for OSPF Networks. ComputerNetworks 48(4) (July 2005)

[7]. Kvalbein, A., et al.: Post-Failure Routing Performance with Multiple Routing Configurations. In: Proc. IEEE (2007)

[8]. Zhang, C., et al.: On Optimal Routing with Multiple Traffic Matrices. In: Proc. IEEE INFOCOM (2005)

[9.] Menth, M., Martin, R.: Network Resilience through Multi-topology Routing. In: Proc International Workshop on Design of Reliable Communication Networks (DRCN) (2005)

[10]. Asgari, H., et al.: Scalable Monitoring Support for Resource Management and Service Assurance. IEEE Network Magazine,2004

[11]. D. 0. Awduche, 1. Malcolm, J. Agogbua, M. ODell, and J. McManus, "Requirements for traffic engineering ove MPLS," Network Working Group, Request for Comments! ht tp : / / search. iet f . org/ rf c/rf c2702. txt, Septembei 1999

[12]. Sridharan, A., et al.: Achieving Near-Optimal Traffic Engineering Solutions for Current OSPF/ISIS Networks. IEEE/ACM Transactions on Networking 13(2) (April 2005)

[13] Menth, M., Martin, R.: Network Resilience through Multi-topology Routing. In: Proc. International Workshop on Design of Reliable Communication Networks (DRCN) (2005)

[14]. Asgari, H., et al.: Scalable Monitoring Support for Resource Management and Service Assurance. IEEE Network Magazine,2009 